\title{
ESTUDO DA MODELAGEM DA PRODUÇÃO DE CAROTENOIDES POR Sporidiobolus salmonicolor CBS 2636 EM BIORREATOR
}

\author{
M. U. JANECZKO ${ }^{1}$, J. KILIAN ${ }^{1}$, A. B. J. SOARES ${ }^{2}$ e M. B. A. SOARES ${ }^{1}$ \\ ${ }^{1}$ Universidade Regional Integrada do Alto Uruguai e das Missões, Departamento de Ciências \\ Agrarias \\ ${ }^{2}$ Universidade Regional Integrada do Alto Uruguai e das Missões, Departamento de Engenharias e \\ Ciências da Computação \\ E-mail para contato: alvarado@uricer.edu.br
}

\begin{abstract}
RESUMO - Alguns micro-organismos estão sendo estudados como potenciais de bioprodução de carotenoides e podem ser utilizados na indústria de alimentos. $\mathrm{O}$ desenvolvimento do modelo matemático permitirá descrever o processo e a sua a otimização proporcionará melhorias na produtividade do metabolismo microbiano, através da manipulação dos parâmetros físico-químicos e nutricionais podendo alterar significativamente o rendimento do produto de interesse. O presente trabalho visa o estudo da modelagem matemática e simulação para a produção de carotenoides em biorreator de batelada. Foram propostos os modelos de inibição pelo substrato, inibição pelo produto e inibição pelo substrato e produto para a bioprodução de carotenóides por Sporidiobolus salminocolor CBS 2636 em meio sintético nas condições otimizadas do processo a $25^{\circ} \mathrm{C}, \mathrm{pH} 4,0,180 \mathrm{rpm}$ e $80 \mathrm{~g} / \mathrm{L}$ de substrato. Os modelos descreveram o processo, podendo ser considerados satisfatórios. O modelo com inibição pelo produto obteve menores desvios ao redor de $16 \%$ e um $\mathrm{R}$ maior a 0,92 . O modelo poder ser considerado como uma ferramenta válida para a predição e concepção de um processo de produção de carotenóides.
\end{abstract}

\section{INTRODUÇÃO}

Devido à conscientização de consumidores, a busca por alimentos saudáveis tem-se incrementado, e nessa classe de alimentos podemos citar os prebióticos, como alimentos funcionais, destacando-se a demanda por carotenóides na indústria alimentar. Os carotenóides formam um dos mais importantes grupos de pigmentos naturais amplamente utilizados na indústria de alimentos. Por isso a modelagem de bioprocessos vem sido utilizada amplamente para a otimização das condições operacionais visando aumentar a sua produção.

Comercialmente, os carotenóides são usados como corantes alimentícios e em suplementos nutricionais. No Brasil, a indústria de alimentos representa cerca de $4 \%$ do PIB e 30 $\%$ do total das exportações. Hoje, os carotenóides utilizados industrialmente são obtidos por via química ou extração de plantas e/ou algas, mas devido à preocupação com o uso de aditivos químicos em alimentos, há interesse nos carotenóides obtidos naturalmente por processos 


\section{9 a 22 de outubro de 2014 \\ Florianópolis/SC}

biotecnológicos. Embora os corantes sejam considerados essenciais à indústria de alimentos, podendo ser obtidos através de fontes naturais, este campo ainda não é bem explorado, em particular os produzidos por micro-organismos.

Muitos micro-organismos já estão sendo estudados como potenciais de bioprodução. Além disso, a produção de carotenóides também tem sido feita por processos biotecnológicos, utilizando produtos agrícolas de baixo custo e resíduos como soro de queijo (Aksu e Eren, 2005, Valduga et al., 2009), mosto de uva (Buzzini e Martini, 1999), derivados do processamento da cana-de-açúcar (Fontana et al., 1996; Valduga et al., 2008), do processamento de milho (Valduga et al., 2008); e da produção de biodiesel (Saenge et al., 2011) como forma de reduzir os custos de produção e a emissão destes subprodutos industriais e agroindustriais no meio ambiente, pois são ricos em açúcares e matéria orgânica.

O estudo da modelagem matemática permite prever o comportamento dinâmico e estacionário do processo, inclusive em condições não testadas empiricamente, possibilitando a determinação de condições operacionais economicamente ótimas do sistema, avaliando o projeto e o ajuste de algoritmos de controle, no qual o modelo matemático formulado passa a ser parte integrante do mesmo (Bonomi e Schmidell, 2001; Ingham et al., 2007). Uma etapa essencial no desenvolvimento de qualquer modelo é a formulação das equações de balanço de massa e energia, podendo-se incluir relações adicionais que representem: as taxas de reação química, as taxas de transferência de calor e massa e alterações de propriedades do sistema. A combinação destas relações constitui a base para a descrição quantitativa do processo e compreende o modelo matemático básico. O modelo resultante pode variar de um simples caso de equações até modelos de grande complexidade. Uma das habilidades da modelagem é, portanto, obter o modelo mais simples possível que seja capaz de uma representação realista do processo (Ingham et al., 2007).

A otimização dos processos biotecnológicos de produção é um tema de grande importância. Na bioprodução, qualquer melhoria alcançada na produtividade do metabolismo microbiano, em geral, através da manipulação dos parâmetros físico-químicos e nutricionais pode alterar significativamente o rendimento do produto de interesse. Neste sentido o presente trabalho tem como objetivo o estudo da modelagem matemática do processo de produção de carotenoides, e a partir deste modelo simular o processo em biorreator.

\section{MATERIAL E MÉTODOS}

Para o modelo matemático foram utilizados os balanços de massa para descrever a cinética de crescimento, o consumo de substrato e a produção de carotenóides para um reator de batelada segundo as equações 1 a 3. Para a determinação da velocidade de crescimento foram utilizados três modelos diferentes segundo as equações 4 a 6 . Os parâmetros cinéticos utilizados foram os determinados previamente por Schwartz (2010). 


$$
\begin{aligned}
& \frac{d S}{d t}=-\left(\frac{\mu}{Y_{X / S}}+m_{s}\right) X \\
& \frac{d X}{d t}=\mu X \\
& \frac{d P}{d t}=(\alpha \mu+\beta) X
\end{aligned}
$$

Inibição pelo substrato

$$
\mu=\frac{\mu_{\max } \cdot S}{K_{s}+S+\frac{S^{2}}{K_{i}}}
$$

Inibição pelo produto

$$
\mu=\frac{\mu_{\max } \cdot S}{K_{s}+S}\left(1-\frac{P}{K_{p}}\right)^{\lambda}
$$

Inibição pelo substrato e produto

$$
\mu=\frac{\mu_{\max } \cdot S}{K_{s}+S+\frac{S^{2}}{K_{i}}}\left(1-\frac{P}{K_{p}}\right)^{\lambda}
$$

\section{RESULTADOS E DISCUSSÃO}

Para as simulações foi utilizado o programa do MATLAB. Os valores das constantes cinéticas foram os obtidos previamente por Schwartz (2010) e o modelo matemático a partir das equações 1 a 3, onde foram utilizados diferentes modelos de velocidade de crescimento.

Os parâmetros cinéticos foram re-estimados obtendo-se valores para os modelos com inibição pelo substrato, inibição pelo produto e inibição pelo substrato e produto como é mostrado na Tabela 1. Os resultados mostram que dependendo do modelo matemático, os parâmetros cinéticos obtiveram-se maiores reajustes. 
Tabela 1 - Parâmetros cinéticos para os diferentes modelos matemáticos para a produção de carotenóides.

\begin{tabular}{lccc}
\hline Parâmetros & Inibição pelo substrato & Inibição pelo produto & $\begin{array}{c}\text { Inibição pelo substrato e } \\
\text { produto }\end{array}$ \\
\hline$\mu_{\max , \mathrm{h}}{ }^{-1}$ & 0,685 & 1,56 & 1,396 \\
$\mathrm{~K}_{\mathrm{s}}, \mathrm{g} / \mathrm{L}$ & 1691,18 & 1681,28 & 2081,18 \\
$\mathrm{~K}_{\mathrm{i}}, \mathrm{g} / \mathrm{L}$ & 10,12 & - & 134,12 \\
$\mathrm{Y}_{\mathrm{xs}}, \mathrm{g} / \mathrm{g}$ & 0,45 & 0,28 & 0,31 \\
$\mathrm{~m}_{\mathrm{s}}, \mu \mathrm{g} / \mathrm{g} \cdot \mathrm{h}$ & 0,02 & 0,008 & 0,007 \\
$\alpha, \mu \mathrm{g} / \mathrm{g}$ & 500 & 500 & 500 \\
$\beta, \mu \mathrm{g} / \mathrm{g} \cdot \mathrm{h}$ & 40 & 40 & 40 \\
$\lambda$ & - & 3,1 & 3,0 \\
$\mathrm{~K}_{\mathrm{p}}, \mu \mathrm{g} / \mathrm{L}$ & - & 0,04 & 0,06 \\
\hline
\end{tabular}

As predições para concentrações de substrato, biomassa e produção de carotenóides foram comparadas com os dados experimentais (Schwartz, 2010). Na tabela 2, os dados mostrados indicam o melhor ajuste para o modelo aos dados experimentais com desvios ao redor de $16 \%$, em relação ao total de carotenóides produzidos após $100 \mathrm{~h}$ de fermentação. Pelos dados da tabela verifica-se que para o modelo com inibição de produto e inibição de produto e substrato apresenta uma maior produção de carotenoides, indicando que possivelmente possa existir alguma reação que não está prevista no modelo.

Tabela 2 - Produção de carotenóides utilizando diferentes modelos matemáticos após 100 h de fermentação.

\begin{tabular}{|c|c|c|c|}
\hline & rimental & Model predictions & RED \\
\hline \multicolumn{4}{|c|}{ Inibição pelo substrato } \\
\hline $\mathrm{S}(\mathrm{g} / \mathrm{L})$ & 38,10 & 38,27 & 0,44 \\
\hline X (g/L) & 13,35 & 14,69 & 9,12 \\
\hline Caro $(\mu \mathrm{g} / \mathrm{L})$ & 3426 & 3340 & 2,57 \\
\hline \multicolumn{4}{|c|}{ Inibição pelo produto } \\
\hline $\mathrm{S}(\mathrm{g} / \mathrm{L})$ & 38,10 & 38,91 & 2,08 \\
\hline$X(g / L)$ & 13,35 & 11,48 & 16,29 \\
\hline Caro $(\mu \mathrm{g} / \mathrm{L})$ & 3426 & 4070 & 15,82 \\
\hline \multicolumn{4}{|c|}{ Inibição pelo substrato e produto } \\
\hline $\mathrm{S}(\mathrm{g} / \mathrm{L})$ & 38,10 & 38,48 & 0,98 \\
\hline$X(g / L)$ & 13,35 & 12,95 & 3,09 \\
\hline Caro $(\mu \mathrm{g} / \mathrm{L})$ & 3426 & 4070 & 15,82 \\
\hline
\end{tabular}

S, Substrato; X, Biomassa; Caro, Carotenóides.

RED, Relative error deviation between experimental data and model predictions, $(\%)$. 
As predições feitas pelo modelo e os dados experimentais para concentrações de substrato, biomassa e carotenóides são mostradas na Figura 1, 2 e 3. Como são mostrados na Figura 1. Observa-se que os dados preditos foram similares aos dados obtidos experimentalmente, com valores de $\mathrm{R}$ maiores que 0,85, 0,92 e 0,91 para os modelos de inibição pelo substrato, inibição pelo produto e inibição pelo substrato e produto, respectivamente.

O modelo que apresentaram melhores resultados e representa melhor a cinética de produção de carotenoides foram o modelo de inibição pelo produto e inibição pelo substrato e produto (Figura 2 e 3). Mas se comparamos com os valores de $\mathrm{R}$ o modelo mais adequado serio o modelo matemático com inibição pelo produto.
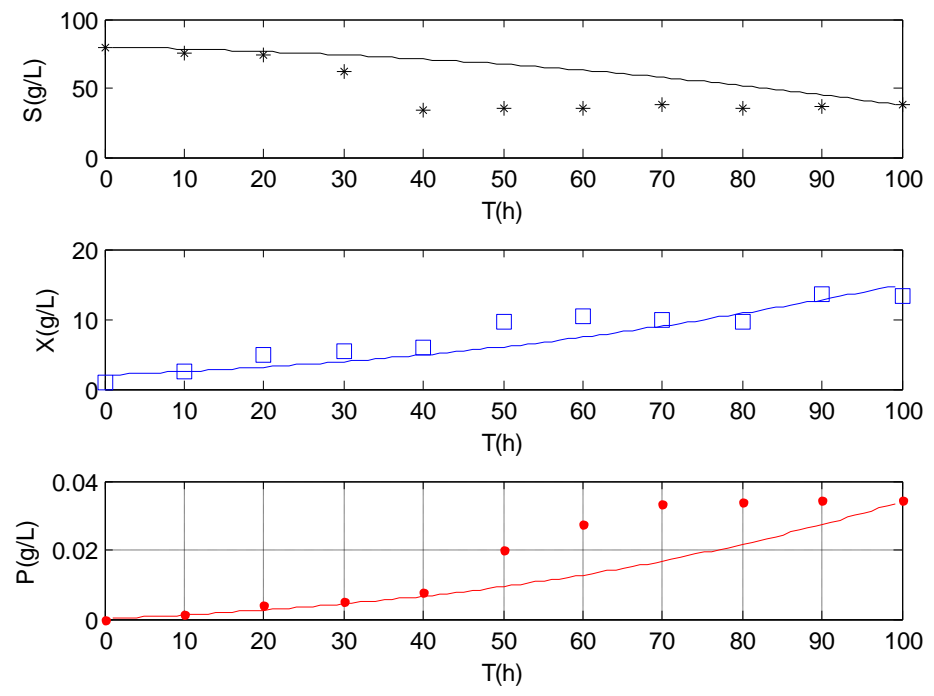

Figura 1 - Comparação da predição do modelo matemático com inibição pelo substrato para a produção de carotenóides, (-) com dados experimentais para cada componente: substrato (*), biomassa $(\square)$, carotenóides $(\bullet)$, nas condições de fermentação a $25^{\circ} \mathrm{C}, \mathrm{pH} 4,0$ e $180 \mathrm{rpm}$, para concentração de substrato de $80 \mathrm{~g} / \mathrm{L}$. 

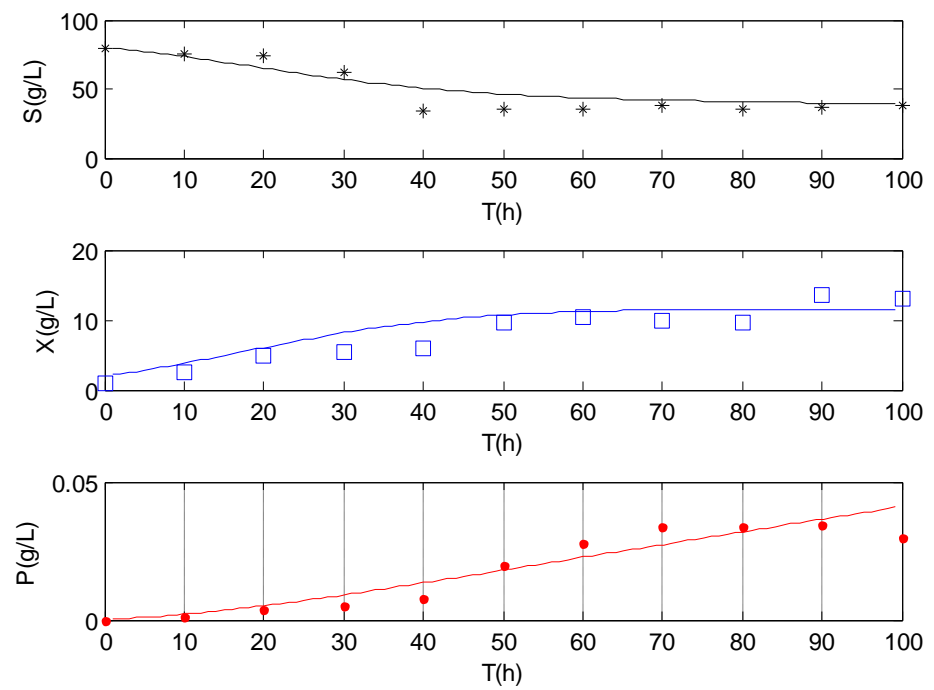

Figura 2 - Comparação da predição do modelo matemático com inibição pelo produto para a produção de carotenóides, $(-)$ com dados experimentais para cada componente: substrato $\left(^{*}\right)$, biomassa ( $\square$ ), carotenóides $(\bullet)$, nas condições de fermentação a $25^{\circ} \mathrm{C}, \mathrm{pH} 4,0$ e $180 \mathrm{rpm}$, para concentração de substrato de $80 \mathrm{~g} / \mathrm{L}$.
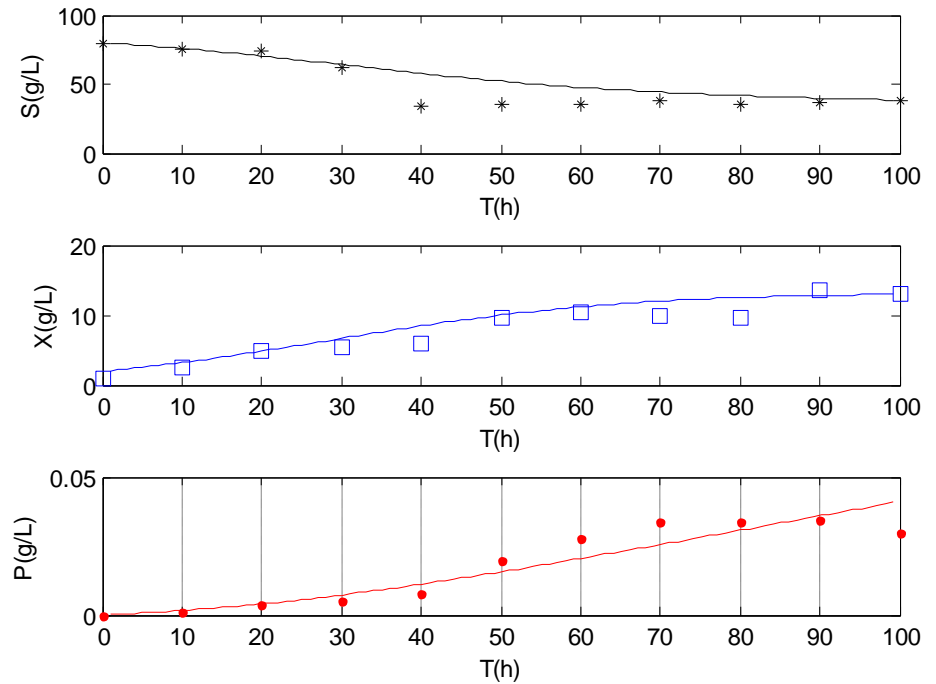

Figura 3 - Comparação da predição do modelo matemático com inibição pelo substrato e produto para a produção de carotenóides, $(-)$ com dados experimentais para cada componente: substrato $(*)$, biomassa $(\square)$, carotenóides $(\bullet)$, nas condições de fermentação a $25^{\circ} \mathrm{C}, \mathrm{pH}$ 4,0 e $180 \mathrm{rpm}$, para concentração de substrato de $80 \mathrm{~g} / \mathrm{L}$. 


\section{CONCLUSSÃO}

De acordo com as previsões dos diferentes modelos matemáticos formulados para descrever o processo fermentativo na produção de carotenóides pela Sporidiobolus salmonicolor CBS 2636 a $25^{\circ} \mathrm{C}$, pH de 4,5, $180 \mathrm{rpm}$ e $80 \mathrm{~g} / \mathrm{L}$ de substrato os resultados podem ser considerados satisfatórios, indicando que o modelo matemático com inibição pelo produto pode ser considerado como uma ferramenta válida para a predição e concepção de um processo de produção de carotenóides.

\section{NOMENCLATURA}

$\mathrm{K}_{\mathrm{i}} \quad$ constante de inibição pelo substrato $(\mathrm{g} / \mathrm{L})$

$\mathrm{K}_{\mathrm{p}} \quad$ constante de inibição pelo produto $(\mu \mathrm{g} / \mathrm{L})$

$K_{\mathrm{s}} \quad$ constante de saturação do substrato $(\mathrm{g} / \mathrm{L})$

$\mathrm{m}_{\mathrm{s}} \quad$ coeficiente de manutenção celular $(\mu \mathrm{g} / \mathrm{g} . \mathrm{h})$

$P \quad$ concentração de produto $(\mathrm{g} / \mathrm{L})$

$\mathrm{t}$ tempo (h)

$X \quad$ concentração de biomassa $(\mathrm{g} / \mathrm{L})$

$Y_{\mathrm{xs}} \quad$ fator de conversão do substrato em células $(\mathrm{g} / \mathrm{g})$

$\alpha \quad$ coeficiente de formação do produto associado ao crescimento $(\mu \mathrm{g} / \mathrm{g})$

$\beta \quad$ formação de produto não associado ao crescimento ( $\mu \mathrm{g} / \mathrm{g} . \mathrm{h}$ )

$\gamma \quad$ termo exponencial para o modelo de inibição pelo produto

$\mu \quad$ velocidade específica de crescimento celular $\left(\mathrm{h}^{-1}\right)$

$\mu_{\max }$ velocidade máxima específica de crescimento celular $\left(\mathrm{h}^{-1}\right)$

\section{REFERENCIAS}

AKSU, Z.; EREN, A. T. Carotenoids production by the yeast Rhodotorula mucilaginosa: Use of agricultural wastes as a carbon source. Process Biochem., v. 40, p. 2985-2991, 2005.

ASSOCIATION OF OFFICIAL ANALYTICAL CHEMISTS - AOAC. Official methods of analysis of the association of the analytical chemists, Washington, 16 ed., 1995.

BONOMI, A.; SCHMIDEELL, W. Processos Fermentativos e enzimáticos. São Paulo: Edgard Blücher Ltda, 2001.

BRITTON, G.; LIAAEN-JENSEN, S.; PFANDER, H. Carotenoids today and challenges for the future. In: Carotenoids. Berlin: Birkhäuser Verlag, p. 13-26, 1995.

BUZZINI, P; MARTINI, A. Production of carotenoids by strains of Rhodotorula glutinis cultured in raw materials of agro-industrial origin. Bioresource Technol., v. 71, p. 41-44, 1999. 
COLET, R. Produção de carotenoides por Sporidiobolus salmonicolor CBS 2636 em biorreator batelada alimentada. Dissertação de Mestrado. Engenharia de Alimentos, URICampus Erechim, 2012.

FONTANA, J. D.; CZECZUGA, B.; BONFIM, T. M. B.; CHOCIAI, M. B.; OLIVEIRA, B. H.; GUIMARÃES, M. F.; BARON, M. Bioproduction of carotenoids: the comparative use of raw sugarcane juice and depolymerized bagasse by Phaffia Rhodozyma. Bioresource Technol., v. 58, p. 121-125, 1996.

INGHAM, J., DUNN. I. J., HEINZLE, E., PRENOSIL, J. E., SNAPE, J. B., Chemical Engineering Dynamics, New York: VHC Publishers INC, 2007.

SAENGE, C.; CHEIRSILP, B.; SUKSAROGE, T.T.; BOURTOOM, T. Potential use of oleaginous red yeast Rhodotorula glutinis for the bioconversion of crude glycerol from biodiesel plant to lipids and carotenoids. Process Biochem., v. 46, p. 210-218, 2011.

TAO, Z.; WANG, G.; XU, X.; YUAN, Y.; WANG, X.; LI, Y. Monitoring and rapid quantification of total carotenoids in Rhodotorula glutinis cells using laser tweezers Raman spectroscopy. FEMS microbial. Lett., v. 314, p. 42-48, 2011.

VAldugA, E., VAlERIO, A., TREICHEL, H., FURIGO JÚNIOR, A., LUCCIO, M. D. Kinetic and Stoichiometric Parameters in the Production of Carotenoids by Sporidiobolus salmonicolor (CBS 2636) in Synthetic and Agroindustrial Media. Appl. Biochem. Biotech., v.157, p. 61- 69, 2009. 